\title{
Atomic excitation during recollision-free ultrafast multi-electron tunnel ionization
}

\author{
W. A. BRYAN ${ }^{1,2 \star}{ }^{2}$, S. L. STEBBINGS ${ }^{1 \dagger}$, J. MCKENNA ${ }^{3}$, E. M. L. ENGLISH ${ }^{1}$, M. SURESH ${ }^{3}$, J. WOOD ${ }^{1}$, \\ B. SRIGENGAN ${ }^{3}$, I. C. E. TURCU², J. M. SMITH², E. J. DIVALL ${ }^{2}$, C. J. HOOKER ${ }^{2}$, A. J. LANGLEY², \\ J. L. COLLIER ${ }^{2}$, I. D. WILLIAMS ${ }^{3 *}$ AND W. R. NEWELL ${ }^{1 *}$
}

\author{
${ }^{1}$ Department of Physics and Astronomy, University College London, Gower Street, London WC1E 6BT, UK \\ ${ }^{2}$ Central Laser Facility, CCLRC Rutherford Appleton Laboratory, Chilton, Didcot, Oxon OX11 0QX, UK \\ ${ }^{3}$ Department of Pure and Applied Physics, Queen's University Belfast, Belfast BT7 1NN, UK \\ ${ }^{\dagger}$ Present address: Department of Physics and Astronomy, University of Southampton, Southampton S017 1BJ, UK \\ "Present address: Cavendish Laboratory, University of Cambridge, Madingley Road, Cambridge CB3 OHE, UK \\ *e-mail: w.bryan@ucl.ac.uk; i.williams@qub.ac.uk; w.r.newell@ucl.ac.uk
}

M odern intense ultrafast pulsed lasers generate an electric field of sufficient strength to permit tunnel ionization of the valence electrons in atoms ${ }^{1}$. This process is usually treated as a rapid succession of isolated events, in which the states of the remaining electrons are neglected ${ }^{2}$. Such electronic interactions are predicted to be weak, the exception being recollision excitation and ionization caused by linearly polarized radiation $^{3}$. In contrast, it has recently been suggested that intense field ionization may be accompanied by a two-stage 'shake-up' reaction ${ }^{4}$. Here we report a unique combination of experimental techniques $^{5-8}$ that allows us to accurately measure the tunnel ionization probability for argon exposed to 50-fs laser pulses. Most significantly for the current study, this measurement is independent of the optical focal geometry ${ }^{7,8}$, equivalent to a homogenous electric field. Furthermore, circularly polarized radiation negates recollision. The present measurements indicate that tunnel ionization results in simultaneous excitation of one or more remaining electrons through shake-up ${ }^{9}$. From an atomic physics standpoint, it may be possible to induce ionization from specific states, and will influence the development of coherent attosecond extreme-ultraviolet-radiation sources ${ }^{10}$. Such pulses have vital scientific and economic potential in areas such as high-resolution imaging of in vivo cells and nanoscale extremeultraviolet lithography.

The noble gas atoms are a natural choice for studying electron dynamics in an ultrafast intense laser pulse, and a number of pivotal experimental studies have established this rich and vibrant field of research, revealing much about the complex nature of photoionization ${ }^{11}$. However, the majority of these studies have used linearly polarized radiation, and used the predictions of tunnelling theory ${ }^{2}$ (generally the AmmosovDelone-Krainov treatment ${ }^{12}$ ) in which the target is confined to the ground state. The current investigation was stimulated by the theoretical work of Eichmann et al. ${ }^{13}$ and Zon et al. ${ }^{4}$, and reveals experimental evidence for a hereto unobserved accompanying excitation mechanism.

In the ultrafast regime, the optical pulse duration is of the order of femtoseconds ( $1 \mathrm{fs}=10^{-15} \mathrm{~s}$ ), and ionization proceeds either by a multiphoton perturbative process ${ }^{14}$ or a nonperturbative strongfield process, generally accepted as being described by tunnel theory: for a recent review see Popov ${ }^{15}$. The present work is concerned purely with strong-field processes (peak intensity greater than approximately $10 \mathrm{TW} \mathrm{cm}^{-2}$, where $1 \mathrm{TW}=10^{12} \mathrm{~W}$ ) where the rate of tunnel ionization is governed by the frequency and strength of the radiation-induced electric field and the binding energy and quantum state of the ion and electron(s).

Immediately following ionization, the 'drive pulse' electron is in a Volkov state ${ }^{16}$ and is initially fully correlated with the parent ion. As the electron is accelerated by the laser field, the electron trajectory is determined by the ellipticity of the field and the phase at which ionization occurred. Electron-impact excitation ${ }^{17}$ or subsequent ionization ${ }^{18}$ can arise in a linearly polarized laser field through the intriguing phenomenon of recollision. Recollision is the key mechanism for attosecond extreme-ultraviolet-pulse generation, as the kinetic energy of the electron is dissipated photonically if the electron is recaptured by the parent ion $^{19}$. However, in the present work, we make recollision events negligible by using circularly polarized light: the absorption of a large number of photons transfers considerable angular momentum to the liberated electron, preventing it from returning to the ionic $\operatorname{core}^{7,20}$, thus the masking effect of recollisional excitation and ionization are negated.

Although strong-field ionization and recollision in ultrafast laser pulses is well documented, minimal theoretical and essentially no experimental studies have investigated the possibility of simultaneous excitation of the parent ion during tunnel ionization. 

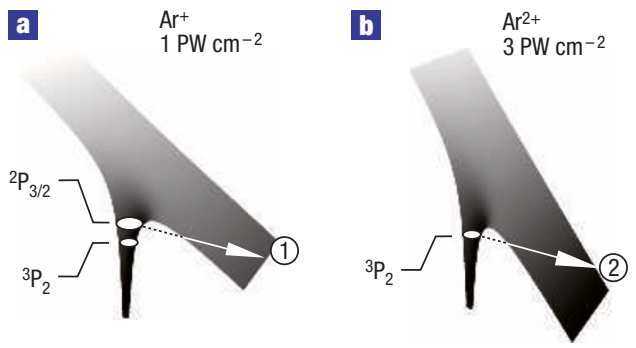

Standard sequentia tunnel ionization

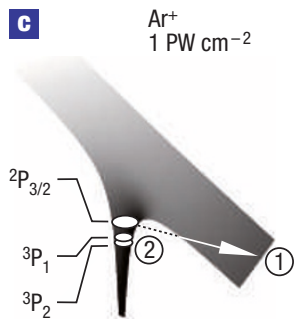

Multi-electron tunnel ionization

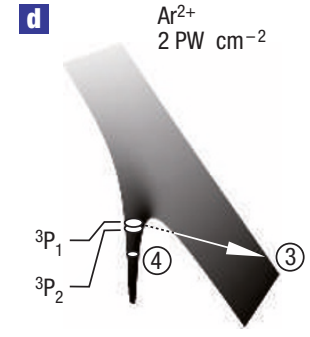

Multi-electron tunnel ionization
Figure 1 Tunnel ionization and resulting excitation mechanisms in the Coulomb potential of argon. Regular sequential tunnel ionization: a, At an intensity of $1 \mathrm{PW} \mathrm{cm}{ }^{-2}$ the ground state $\left({ }^{2} \mathrm{P}_{3 / 2}\right)$ of $\mathrm{Ar}^{+}$has a significant probability to tunnel ionize (1). $\mathbf{b}$, As the intensity is increased to $3 \mathrm{PW} \mathrm{cm}{ }^{-2}$, the ground state $\left({ }^{3} \mathrm{P}_{2}\right)$ of $\mathrm{Ar}^{2+}$ can ionize (2). Considering multi-electron processes, the tunnel ionization is significantly influenced by excitation. c, As the ${ }^{2} \mathrm{P}_{3 / 2}$ electron tunnels from the $\mathrm{Ar}^{+}$ ion (1), a significant population is transferred from the ${ }^{3} \mathrm{P}_{2}$ to ${ }^{3} \mathrm{P}_{1}$ excited state in the $\mathrm{Ar}^{2+}$ ion (2). $\mathbf{d}$, A lower intensity $\left(2 \mathrm{PW} \mathrm{cm}{ }^{-2}\right)$ is then required to ionize the population in the ${ }^{3} \mathrm{P}_{1}$ state (3), whereas the ${ }^{3} \mathrm{P}_{2}$ state ionizes at $3 \mathrm{PW} \mathrm{cm}{ }^{-2}$ (b). The generation of $\mathrm{Ar}^{2+}$ can then further excite the $\mathrm{Ar}^{3+}$ ion (4). We measure the dependence of the probability of ionization on the laser intensity and charge state.

In the case of multiphoton ionization, resonant excitation processes have been identified ${ }^{21-23}$, however in the present work, the intensity of the laser field is sufficient that tunnel ionization occurs with a dramatically enhanced probability. The contemporary work of Zon ${ }^{4,24,25}$ invoked the idea of 'inelastic tunnelling' whereby the parent ion is left in an excited state following the ionization of one of $N$ identical valence electrons. Excitation is through 'shake-up', first used by Carlson ${ }^{9}$ to explain single ultraviolet photon absorption leading to the ionization of a first and the excitation of a second electron. The ionization event diabatically distorts the electron wavefunctions, resulting in the excitation of a bound second electron. Using this concept, Zon et al. ${ }^{4,24}$ and Kornev et al. ${ }^{25}$ derived a general expression for the rate of tunnel ionization (TI) of an atom with simultaneous excitation of the lowest-lying ionic states. This treatment relies on the sudden approximation (SA), which is valid provided accessing the excited states in $\mathrm{Ar}^{(q+1)+}$ requires considerably less energy than the ionization potential of $\mathrm{Ar}^{q+}$. Kornev et al. ${ }^{25}$ show that although this has negligible influence on the ionization probability for the $\mathrm{Ar}^{q+} \rightarrow \mathrm{Ar}^{(q+1)+}$ process, the creation of excited states in the product $\mathrm{Ar}^{(q+1)+}$ ion strongly influences the $\mathrm{Ar}^{(q+1)+} \rightarrow \mathrm{Ar}^{(q+2)+}$ ionization probability. The consequence of this mechanism is illustrated in Fig. 1. Furthermore, by allowing for all combinations of excitation and ionization, the significant role of simultaneous excitation and ionization during tunnelling in the laser field

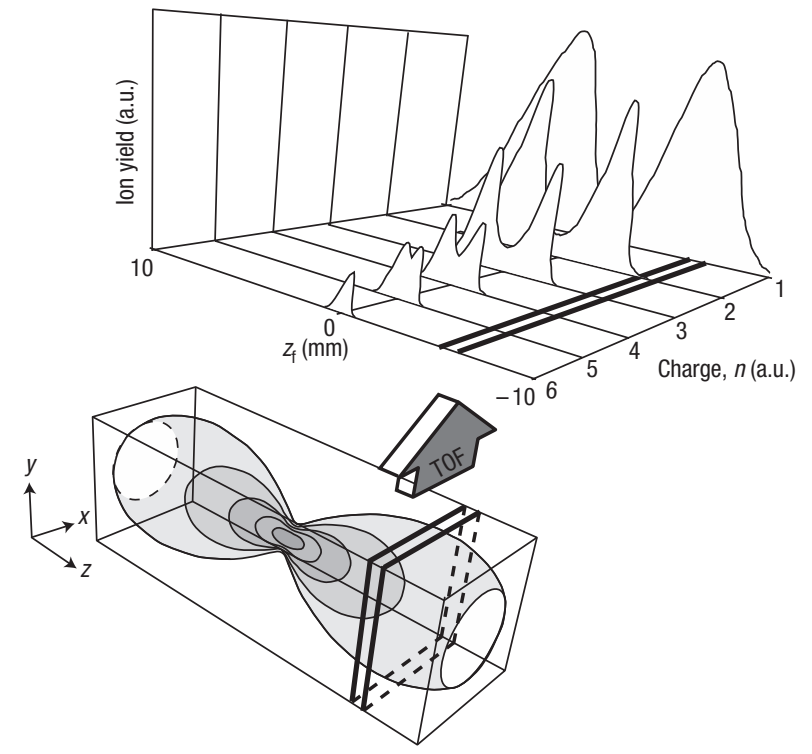

Figure 2 Illustration of the ISS technique $e^{5-8}$ and measured ion yield data. As the laser focus (bottom) is translated with respect to the narrow detection aperture of the TOFMS (slot perpendicular to $z_{\mathrm{f}}$, the distance from the focus), the ion yield for all charge states of $\operatorname{Ar}^{q+}(q=1-6)$ is measured. The laser pulse propagates in the $z$ direction, and the focus is translated parallel to this axis.

is highlighted. To distinguish from standard sequential tunnel ionization (TI), we refer to such processes as multi-electron tunnel ionization (METI).

The analogous process of 'shake-off' resulting in further ionization rather than discrete excitation was originally proposed a decade ago by Fittinghoff et al. ${ }^{26}$ in an attempt to quantify the many orders of magnitude discrepancy between the predicted sequential TI yield and the observed $\mathrm{He}^{2+}$ ion yield by laser radiation ${ }^{27}$. It has been through careful experiments using linear and circular polarization (see for example the work of Guo et al. ${ }^{28}$ ) and the cold-target recoil-ion-momentum spectroscopy technique ${ }^{17,18}$ that recollision has finally been accepted as the mechanism responsible. More recently, the shake-off ionization of helium exposed to ultrafast laser pulses has been investigated theoretically using the $S$-matrix technique. Becker and Faisal calculated the ratio of the rates of shake-off to recollision, and demonstrated that shake-off was many orders of magnitude weaker ${ }^{29}$. As a result, the presence of shake-off in the strong-field ionization of atoms has generally been dismissed for the case of linear polarization. In the present work, we propose that the relevance of shake-up excitation be re-examined in the case of argon, given that the validity of the SA is significantly different from the case of helium discussed above. The far lowerlying excited states in $\mathrm{Ar}^{q+}(q=1-6)$ are far more readily accessed during $\mathrm{TI}$, in contrast to the extremely high excitation energies of $\mathrm{He}^{+}$, irreconcilable with the SA.

In general, previous experimental measurements of ion yield as a function of laser intensity are a convolution of the ion signal with the focal volume producing the signal. By simply changing the energy of the laser pulse, the spatial distribution of laser intensity also changes. Frequently, the complexity of this situation is compounded by diffraction associated with the spatial profile of the laser ${ }^{7}$; a direct comparison with theory is thus impossible without introducing the specific experimental geometry. A novel solution to this 'volume variation' problem, referred to as intensity selective scanning (ISS), was demonstrated by Van Woerkum ${ }^{5}$; 


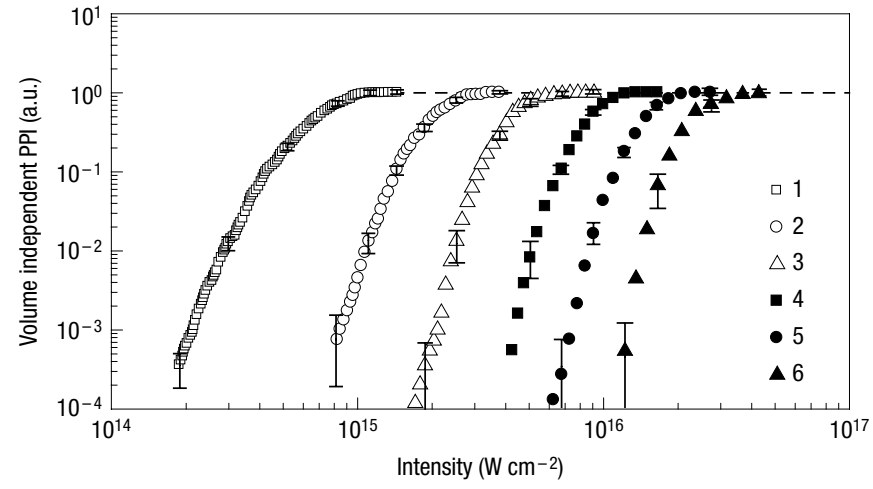

Figure $3 \mathrm{PPI}$ to $\mathrm{Ar}^{q+}(q=1-6)$ as a function of spatially independent laser intensity. As circularly polarized radiation is used, this data is free from the influence of recollision effects. The partial probability is recovered from the ISS data in Fig. 2 using the method described in ref. 7. The uncertainty is estimated by calculating the statistical deviation in the recorded average time-of-flight signal, and the upper and lower confidence interval corresponding to $1 \mathrm{~s}$.d. was propagated through the deconvolution procedure. The dashed line indicates a PPI of unity, whereby ionization is saturated.

the relevance of ISS is described in the Methods section, and is illustrated in Fig. 2. The ion yield recorded at each position $z_{\mathrm{f}}$, the distance of the spectrometer axis from the focus, still inherently depends on the intensity distribution in the $(x, y)$ plane. Two numerical methods have been proposed for removing spatial integration over each 'slice' through the focal volume: Walker et al. ${ }^{5}$ described a deconvolution routine, and Goodworth et al. ${ }^{8}$ proposed a tomographic method. Both recover focal geometry-independent probabilities of ionization; the authors have extended the former to experimentally realistic non-gaussian focal conditions ${ }^{7}$.

In Fig. 2, we present a measurement of the spatial distribution of ions generated when argon is exposed to circularly polarized 50 -fs pulses over the intensity range $100 \mathrm{TW} \mathrm{cm}^{-2}$ to $100 \mathrm{PW} \mathrm{cm}^{-2}$. This experiment was carried out using the ASTRA Laser Facility at the Rutherford Appleton Laboratory, as detailed in the Methods section. The $\operatorname{Ar}^{q+}(q=1-6)$ ion yield was measured as the laser focus was translated by a computer-driven motion stage, exposing the detector to different 'slices' through the focal volume: the spatial selectivity of the time-of-flight mass spectrometer (TOFMS) (grey arrow) is indicated by the narrow slice in the $(x, y)$ plane.

The partial probability of ionization (PPI) to $\mathrm{Ar}^{q+}(q=1-6)$ shown in Fig. 3 is recovered from the ISS data (Fig. 2) using the technique pioneered by the authors ${ }^{7}$. Importantly, we totally remove any instrument dependence, including the enhancement of detector gain with charge state. The distinction 'partial' is necessary as the deconvolution method is only valid at intensities below saturation (as indicated by the dashed line), irrespective of diffraction effects. The saturation intensity is defined as described in the Methods section. The PPI for each ionization state (Fig. 3) can be directly recast in terms of the universal conserved probability of ionization (CPI), which embraces all charge states. The CPI for each charge state is presented in Fig. 4, and is discussed in the Methods section.

The spatially homogenous intensity-dependent predictions of Kornev et al. ${ }^{25}$ are perfect for direct comparison with the measured CPI data. Such a comparison is presented in Fig. $4 a-f$, where the data points are the present measurements, the thin lines are the calculated probabilities of producing $\mathrm{Ar}^{q+}$ from the $\mathrm{Ar}^{(q-1)+}$ ground state only (sequential TI), and the thick lines are the METI predictions including shake-up. Note that the experimental CPI
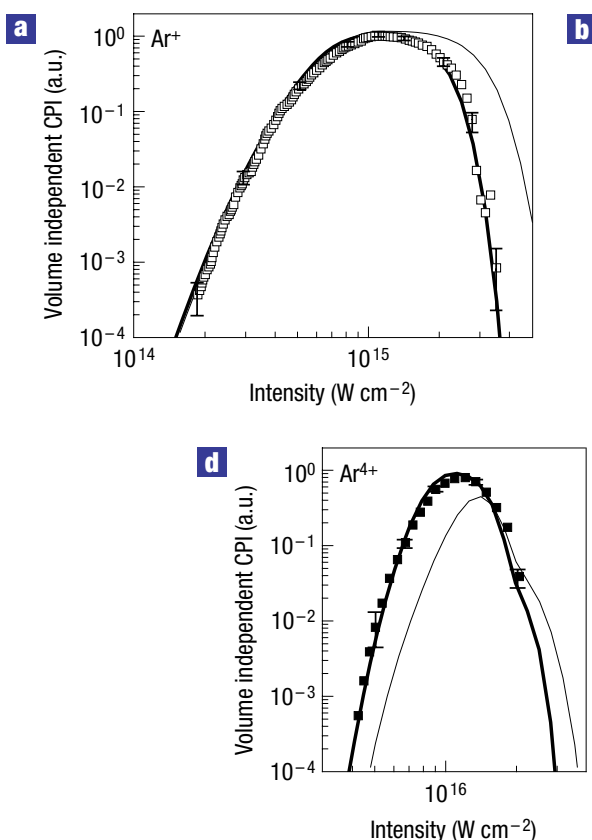
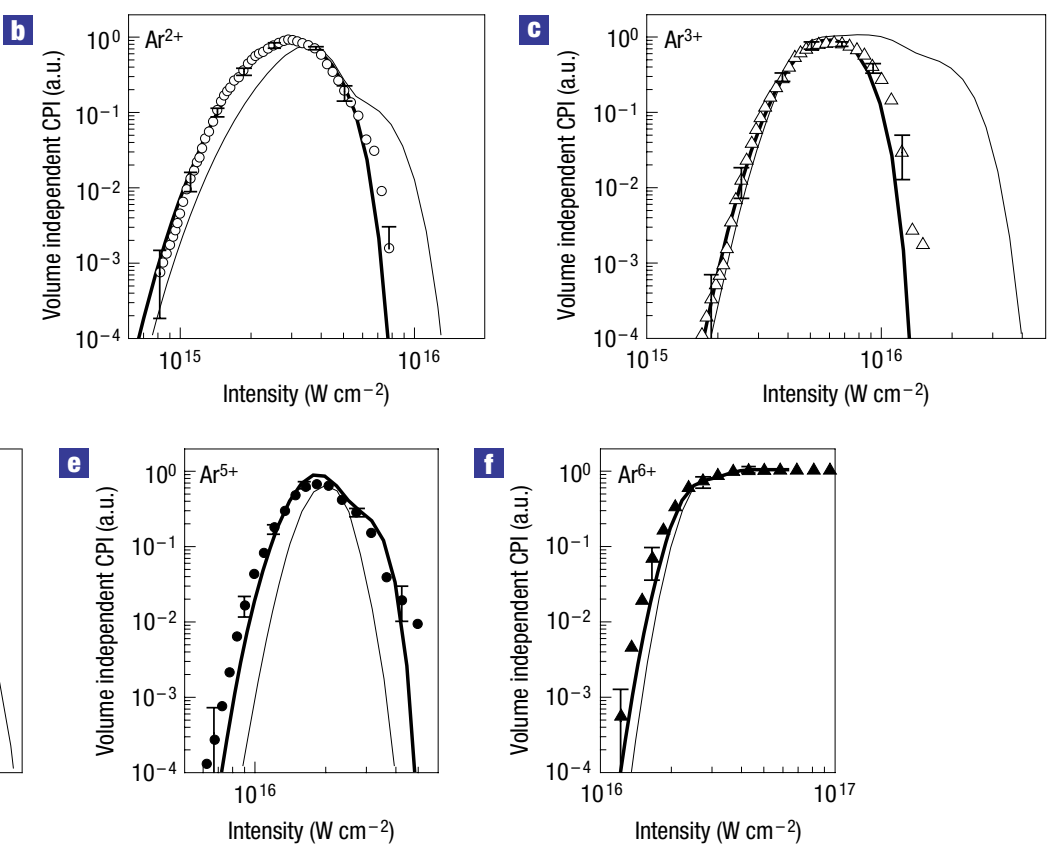

Figure $4 \mathrm{CPI}$ to $\mathrm{Ar}^{q+}(q=1-6)$ as a function of spatially independent laser intensity. $\mathbf{a}, \mathrm{Ar}^{+}$. b, $\mathrm{Ar}^{2+}$. c, $\mathrm{Ar}^{3+}$. d, $\mathrm{Ar}^{4+}$. e, $\mathrm{Ar}^{5+}$. $f, \mathrm{Ar}^{6+}$. Data points: current results derived $^{7}$ from the data in Fig. 3. Thin lines: theoretical predictions ${ }^{25}$ of sequential multiple TI from ionic ground states only, equivalent to Ammosov-Delone-Krainov theory ${ }^{12}$. Thick lines: full METI treatment allowing for shake-up excitation during tunnelling. The uncertainty in the CPI is estimated by propagating the uncertainty in the PPI through the deconvolution procedure. 
has been normalized to the theoretical predictions through the rising edge of the $\mathrm{Ar}^{+} \mathrm{CPI}$; this first ionization stage will not be influenced by excitation. Figure 4 clearly highlights the significant difference between theory and experiment when only sequential TI from the ground state is considered (thin line), lying generally well outside the experimental uncertainty. This is illustrated in Fig. 4 by representative error bars. However, when shake-up is included in the METI prediction, an excellent agreement is found for all charge states as indicated by the thick lines in Fig. 4. Although other possible excitation mechanisms could also be considered, for example the 'way-out' excitation, whereby the departing electron scatters from another bound electron ${ }^{3}$, the observed agreement is strongly supportive of the shake-up model. This benefits from the repeated diabatic distortion of the valence electronic wavefunctions during tunnel ionization at high intensities over a number of optical cycles at intensities greater than $1 \mathrm{PW} \mathrm{cm}^{-2}$. This is an important contrast to single photon shake-off ${ }^{30}$, as the cumulative influence of the oscillatory laser field needs to be considered.

To conclude, we have recorded for the first time strong evidence for the presence of considerable atomic excitation during tunnel ionization by a $790-\mathrm{nm} 50$-fs circularly polarized laser pulse focused to intensities in excess of $100 \mathrm{TW} \mathrm{cm}^{-2}$. Such an observation is made without the need to embrace recollision processes. The significance of the agreement between our experimental observations and recent theoretical predictions indicate that excitation during ionization must be considered irrespective of recollision processes, referred to as multi-electron tunnel ionization. Such excitation will also occur in a linearly polarized laser field, and is expected to be even more influential in a 5-fs few-cycle laser pulse ${ }^{25}$. As the proposed method for generating intense bursts of extreme-ultraviolet radiation relies on accurately controlling high harmonic generation in few-cycle intense laser fields ${ }^{19}$, excitation during tunnelling has a major bearing on the emerging field of optical attosecond physics. Accurate manipulation of the liberated electron motion during the infrared 'drive pulse' and the occupation of initial and transient electronic states will allow tuning of the spectrum of the extremeultraviolet pulse.

\section{METHODS}

\section{INTENSITY SELECTIVE SCANNING}

We are interested in ionization and excitation processes that saturate at intensities less than $100 \mathrm{PW} \mathrm{cm}{ }^{-2}$, therefore the 30-mJ 790-nm 50-fs laser pulses generated by the ASTRA Laser Facility (UK) need only be softly focused ( $\mathrm{f} / 11$ optics) to generate a peak intensity in excess of $100 \mathrm{PW} \mathrm{cm}^{-2}$. Indeed, multiple ionization occurs over tens of millimetres. By measuring the $\mathrm{Ar}^{q+}$ $(q=1-6)$ ion yield with a tightly apertured $(250 \mu \mathrm{m})$ ion TOFMS, only those ions generated within a narrow spatial (and therefore intensity) window are detected. Then, as the focusing optic is translated, the spectrometer is only exposed to ions generated by a well-defined laser intensity ${ }^{5-8,25}$, as illustrated in Fig. 2. Throughout this measurement, the argon gas pressure is low enough to avoid space-charge effects, tested by repeating the ISS measurements as a function of target gas pressure. The narrowing of the spatial distribution of ions as a function of $z_{\mathrm{f}}$ for increasing $n$ is representative of the increasing ionization potential, where the highest ionization state presented $\left(\mathrm{Ar}^{6+}\right)$ is peaked at $z_{\mathrm{f}}=0 \mathrm{~mm}$ where the intensity is at a maximum.

\section{DECONVOLUTION OFANON-GAUSSIAN FOCUS}

The ion yield measured by intensity selective scanning depends very specifically on the spatial distribution of intensity within our focal volume. To make this measurement more universal, we remove this dependence through a deconvolution technique ${ }^{7}$, requiring the measured ion yield and theoretical on-axis intensity as a function of focal position as inputs. The resulting PPI is independent of the variation of the signal-producing volume. We are also able to account for the unavoidable diffraction of the laser pulse ${ }^{7}$ by solving the
Huygens-Fresnel diffraction integral. The PPI is equivalent to the response of a single atom to a spatially infinite laser focus, directly comparable to theoretical predictions, which tend to be presented in terms of a homogenous laser field. The PPI results are valid up to saturation, however at higher intensities the deconvolution breaks down, and the PPI is defined as unity. By measuring the first derivative of the PPI, the saturation intensity is defined as the point at which the gradient falls below the equivalent uncertainty on the PPI. The uncertainty is estimated by calculating the statistical deviation in the recorded average time-of-flight signal, and the upper and lower confidence interval corresponding to 1 s.d. was propagated through the deconvolution procedure. Furthermore, the probability at which saturation occurs depends on the quantum efficiency of the detector, which varies with charge state. The deconvolution method can be used to remove this dependence by normalizing the saturation PPI to unity.

\section{CONSERVATION OF PROBABILITY}

Consider the $\operatorname{PPI}(n)$ for $n=1,2$ as shown in Fig. 3. At low intensity, $\operatorname{PPI}(1)$ is small, and increases with intensity up to saturation at an intensity of $1 \mathrm{PW} \mathrm{cm}{ }^{-2}$. However, as is often the case in atomic ionization, $\mathrm{PPI}(2)$ is non-zero at this intensity. The condition for conserving probability is that the sum of probabilities is less than unity below the saturation intensity of PPI(1) or equal to unity above the saturation intensity of PPI(1), thus at an intensity greater than $\sim 1 \mathrm{PW} \mathrm{cm}{ }^{-2}$, the conserved probability of ionization to $\mathrm{Ar}^{+}$, CPI(1) must be less than unity as PPI(2) is non-zero. This definition is extended to an $N$ electron system in the present work. The uncertainty in the $\mathrm{CPI}$ is estimated by propagating the uncertainty in the PPI through the deconvolution procedure.

\section{Received 16 November 2005; accepted 13 April 2006; published 21 May 2006}

\section{References}

1. Augst, S., Strickland, D., Meyerhofer, D. D., Chin, S. L. \& Eberly, J. H. Tunneling ionization of noble gases in a high-intensity laser field. Phys. Rev. Lett. 63, 2212-2215 (1989).

2. Keldysh, L. V. Ionization in the field of a strong electromagnetic wave. Sov. Phys. JETP 20, 1307-1314 (1965).

3. Becker, A., Dorner, R. \& Moshammer, R. Multiple fragmentation of atoms in femtosecond laser pulses. J. Phys. B 38, S753-S772 (2005)

4. Zon, B. A. Tunnelling ionization of atoms with excitation of the core. JETP 91, 899-904 (2000).

5. Walker, M. A., Hansch, P. \& Van Woerkom, L. D. Intensity-resolved multiphoton ionization: circumventing spatial averaging. Phys. Rev. A 57, R701-R704 (1998).

6. El-Zein, A. A. A. et al. A detailed study of multiply-charged ion production within a high intensity laser focus. Phys. Scr. T 92, 119-121 (2001).

7. Bryan, W. A. et al. Geometry- and diffraction-independent ionization probabilities in intense laser fields: probing atomic ionization mechanisms with effective intensity matching. Phys. Rev. A 73, 013407 (2006).

8. Goodworth, T. R. J., Bryan, W. A., Williams, I. D. \& Newell, W. R. Reconstruction of atomic ionization probabilities in intense laser fields. J. Phys. B 38, 3083-3090 (2005).

9. Carlson, T. A. Double electron ejection resulting from photo-ionization in the outer-most shell of $\mathrm{He}$ $\mathrm{Ne}$, and Ar, and its relationship to electron correlation. Phys. Rev. 156, 142-149 (1967).

10. Baltuška, A. et al. Attosecond control of electronic processes by intense light fields. Nature $\mathbf{4 2 1}$ 611-615 (2003).

11. Larochelle, S., Talebpour, A. \& Chin, S. L. Non-sequential multiple ionization of rare gas atoms in a ti:sapphire laser field. J. Phys. B 31, 1201-1214 (1998).

12. Ammosov, M. V., Delone, N. B. \& Krainov, V. P. Tunnel ionization of complex atoms and of atomic ions in an alternating electromagnetic field. Sov. Phys. JETP 64, 1191-1194 (1986).

13. Eichmann, U., Dörr, M., Maeda, H., Becker, W. \& Sandner, W. Collective multielectron tunneling ionization in strong fields. Phys. Rev. Lett. 84, 3550-3554 (2000).

14. Delone, N. B. \& Krainov, V. P. Multiphoton Processes in Atoms 2nd edn (Springer, Berlin, 2000).

15. Popov, V. S. Tunnel and multiphoton ionization of atoms and ions in a strong laser field (Keldysh theory). Physics-Usp. 47, 855-885 (2004).

16. Volkov, D. M. Over a class of solutions of the Dirac equation. Z. Phys. 94, 250-260 (1935).

17. Feuerstein, B. et al. Separation of recollision mechanisms in nonsequential strong field double ionization of Ar: the role of excitation tunneling. Phys. Rev. Lett. 87, 043003 (2001).

18. Moshammer, R. et al. Correlated two-electron dynamics in strong-field double ionization. Phys. Rev. A 65, 035401 (2002).

19. Reider, G. A. XUV attosecond pulses: generation and measurement. J. Phys. D 37, R37-R48 (2004).

20. Suresh, M. et al. Multiple ionization of ions and atoms by intense ultrafast laser pulses. Nucl. Instrum. Methods Phys. Res. B 235, 216-220 (2005).

21. de Boer, M. P. \& Muller, H. G. Observation of large populations in excited states after short-pulse multiphoton ionization. Phys. Rev. Lett. 68, 2747-2750 (1992).

22. Jones, R. R., Schumacher, D. W. \& Bucksbaum, P. H. Population trapping in $\mathrm{Kr}$ and Xe in intense laser fields. Phys. Rev. A 47, 49-52 (1993).

23. Wells, E., Ben-Itzhak, I. \& Jones, R. R. Ionization of atoms by the spatial gradient of the pondermotive potential in a focused laser beam. Phys. Rev. Lett. 93, 023001 (2004)

24. Zon, B. A. Many-electron tunnelling in atoms. JEPT 89, 219-222 (1999).

25. Kornev, A. S., Tulenko, E. B. \& Zon, B. A. Kinetics of multiple ionization of rare-gas atoms in a circularly polarized laser field. Phys. Rev. A 68, 043414 (2003).

26. Fittinghoff, D. N., Bolton, P. R., Chang, B. \& Kulander, K. C. Observation of nonsequential double ionization of helium with optical tunneling. Phys. Rev. Lett. 69, 2642-2645 (1992).

27. Walker, B. et al. Precision measurement of strong field double ionization of helium. Phys. Rev. Lett. 73, 1227-1230 (1994).

28. Guo, C., Li, M., Nibarger, J. P. \& Gibson, G. N. Single and double ionization of diatomic molecules in strong laser fields. Phys. Rev. A 58, R4271-R4274 (1998). 
29. Becker, A. \& Faisal, F. H. M. S-matrix analysis of coincident measurement of two-electron energy distribution for double ionization of He in an intense laser field. Phys. Rev. Lett. 89, 193003 (2002). 30. Levin, J. C. et al. Measurement of the ratio of double-to-single photoionization of helium at $2.8 \mathrm{keV}$ using synchrotron radiation. Phys. Rev. Lett. 67, 968-971 (1991).

\section{Acknowledgements}

This work is funded by the Engineering and Physical Sciences Research Council (EPSRC), UK.

Research studentships are acknowledged by J.W., E.M.L.E. and S.L.S. (EPSRC), J.M. (DEL) and M.S
(IRCEP at QUB). The authors gratefully acknowledge A. S. Kornev and B. A. Zon from Voronezh State University, Russia for fruitful discussions, suggestions and electronically communicating their theoretical data.

Correspondence and requests for materials should be addressed to W.A.B., I.D.W. or W.R.N.

Competing financial interests

The authors declare that they have no competing financial interests.

Reprints and permission information is available online at http://npg.nature.com/reprintsandpermissions/ 